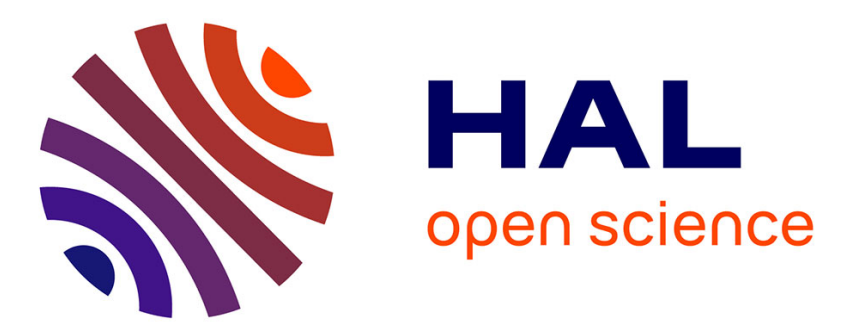

\title{
Robotic assistance for ultrasound elastography providing autonomous palpation with teleoperation and haptic feedback capabilities
}

\author{
Pedro A Patlan-Rosales, Alexandre Krupa
}

\section{- To cite this version:}

Pedro A Patlan-Rosales, Alexandre Krupa. Robotic assistance for ultrasound elastography providing autonomous palpation with teleoperation and haptic feedback capabilities. BioRob 2020 - 8th IEEE RAS/EMBS International Conference for Biomedical Robotics and Biomechatronics, Nov 2020, New York, United States. pp.1-6. hal-02895069

\section{HAL Id: hal-02895069 \\ https://hal.inria.fr/hal-02895069}

Submitted on 9 Jul 2020

HAL is a multi-disciplinary open access archive for the deposit and dissemination of scientific research documents, whether they are published or not. The documents may come from teaching and research institutions in France or abroad, or from public or private research centers.
L'archive ouverte pluridisciplinaire $\mathbf{H A L}$, est destinée au dépôt et à la diffusion de documents scientifiques de niveau recherche, publiés ou non, émanant des établissements d'enseignement et de recherche français ou étrangers, des laboratoires publics ou privés. 


\title{
Robotic assistance for ultrasound elastography providing autonomous palpation with teleoperation and haptic feedback capabilities
}

\author{
Pedro A. Patlan-Rosales ${ }^{1}$ and Alexandre Krupa ${ }^{1}$
}

\begin{abstract}
Detecting stiff tissue using ultrasound elastography has been used as a non-invasive technique in the assessment of important diseases. The real-time estimation of tissue elastic parameters depends on the continuous application of an appropriate palpation motion with an ultrasound probe, which can be achieved through the use of a robotic system. To complement ultrasound elastography and further profit from the information it provides, we propose to give to the clinician the ability to physically feel in real-time during the examination the stiffness of a tissue observed in the elastography image by rendering it with a haptic force feedback, enhancing therefore the capacity of the examiner to detect anomalies. We also propose in our robotic palpation system a teleoperation control of the ultrasound probe for navigation purpose during the tissue examination. Experimental results obtained on an abdominal phantom demonstrated the feasibility of our approach.
\end{abstract}

\section{INTRODUCTION}

Manual palpation is a medical procedure that has been used in diagnosis for centuries, in which the stiffness of the tissue of a patient is felt with the examiner's hands. It allows to recognize changes on the stiffness of the tissue, indicating a possible disease. This practice is non invasive, simple in concept and needs no equipment. However, it requires great expertise and has significant constraints: it provides only qualitative information, it can be affected by the surrounding tissue and it is limited to the tissues within the reach of the examiner's hands. In the literature, we can find several solutions to overcome the limitations of manual palpation, which can be classified by the type of feedback as haptic and pseudo-haptic. Haptic feedback provides examiners with the sensation of touching a tissue through a haptic device. The haptic feedback is usually rendered as force and/or torque by the haptic device. This haptic feedback is of the utmost importance for surgeons, enhancing their abilities to perform teleoperated minimally invasive surgery (TMIS). There are several works where haptic feedback is used as part of assistance systems. Haptic force feedback is commonly generated by sensing the force on the surgical tools, or by adding virtual fixtures to increase the safety during TMIS [1]. Haptic force feedback has also shown benefits in the performance of the suturing process with a surgical robot [2]. A finite element model of a soft tissue was used in a palpation simulator to generate haptic feedback based on the stiffness of the tissue model [3]. More recently, a disposable haptic palpation probe was designed to locate blood vessels during a TMIS [4]. Haptic feedback has also been explored and evaluated in a

\footnotetext{
${ }^{1}$ Pedro A. Patlan-Rosales and Alexandre Krupa are with Univ Rennes, Inria, CNRS, IRISA, campus universitaire de Beaulieu, Rennes 35042, France. alexandre.krupalinria.fr
}

robotic assistance system for needle insertion combined with haptic palpation [5]. Indeed, haptic feedback has shown good performance in palpation, although it still has limitations regarding the deepness of the tissue that it is able to render.

Pseudo-haptic feedback can be seen as a fusion between haptic feedback and other kinds of feedback. Here, we focus more particularly on the combination of haptic and visual feedback. For instance, visual cues have been used to enhance haptic feedback for palpation on a virtual model of soft tissue [6]. The combined feedback method has shown good performance in medical palpation simulators. Medical imaging can also be used to provide visual feedback exploiting its non-invasive capabilities of reaching tissues inside of the body. For instance, ultrasound imaging has been used to visualize the tongue while a subject is speaking, providing feedback for speech therapy [7].

Our work presented in this paper takes place in a research project that focuses on the development of a robotic assistance system for ultrasound elastography (USE). USE has been explored in medicine for the diagnosis of breast tumors [8], liver fibrosis at different stages [9] and prostate cancer [10]. In a previous work [11], we developed a robotic palpation system that consists of an ultrasound probe held by a 6 degrees of freedom (DOF) robot (see Fig. 1a). A force control law was proposed to automatically and continuously apply a quasi-static compression over the tissue with the ultrasound probe in order to obtain the pre- and post-compressed states of the tissue. A method was also proposed to estimate in real-time the elastic parameters as strain values of the observed tissue from the radio frequency (RF) data acquired by the ultrasound probe between two consecutive ultrasound images. In this previous work, the tissue elastic parameters was provided to the examiner by a visual feedback thanks to the display of a color-coded image that represents a 2D elasticity map of the tissue called elastogram. In order to enhance the examiner's perception, we propose in this new work to give him also the ability to physically feel the elasticity of the observed tissue through the use of a haptic device during the tissue examination. Haptic force feedback has been studied before for tissue examination [12] using a position control of a force sensing probe. USE images were also used to provide haptic force feedback with a tissue simulator for ultrasound palpation [13]. However, none of these works use haptic force feedback from USE images during real-time tissue examination. We propose in this paper a solution that provides, via the use of a haptic device, a force feedback to the user directly during the examination. This force is computed from the elastogram live stream generated 
by our robotic palpation system presented in [11].

In addition, we also exploit the haptic device to teleoperate the ultrasound probe for navigation purpose. Therefore, our approach would offer an excellent perspective to enhance the capabilities of the examiner to localize anomalies in the tissues by using a robotic palpation approach with visual and haptic feedback.

In the next section, we briefly recall the force control law used in the robotic palpation system we presented in [11], which generates the compression motion on the tissue that is required for the elastogram estimation. Then, we present in section III the contribution of this paper that concerns the development of two robotic assistance modes for USE. The first mode allows the user to teleoperate the ultrasound probe for changing the ultrasound view while applying the autonomous palpation motion and the second mode provides to the user a force feedback reflecting the tissue elasticity. Experimental results of these assistance modes are then presented in section IV.

\section{PALPATION MOTION TASK - PREVIOUS WORK}

We briefly recall our previous work presented in [11] that concerned the design of a robotic system for soft tissue palpation for real-time elastography imaging. A force control law was proposed to automatically and continuously apply a quasi-static compression over the tissue with an ultrasound probe attached to a 6-DOF robot (see Fig. 1a). This compression process is performed to obtain the preand post-compressed states of the tissue that are required to estimate its elastogram (elasticity map) from radio frequency (RF) data acquired by the ultrasound probe. To obtain this
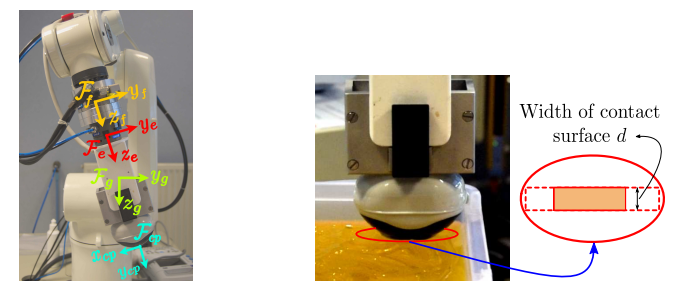

(a) Coordinate frames attached to (b) Width of contact surface the robot. corresponding to the length of the virtual spring.

Fig. 1: Robotic palpation system.

continuous and periodical deformation of the tissue, we designed a force control law that applies a desired force variation along the $y$-axis of the cartesian frame $\mathcal{F}_{c p}$ attached at the bottom of the ultrasound probe (see Fig. 1a) that we defined as,

$$
s_{f}^{*}(k)=\frac{\Delta_{F}}{2}\left[\sin \left(\frac{(4 k-T) \pi}{2 T}\right)+1\right]+F_{0},
$$

where $k$ is the discrete time and $\Delta_{F}$ is the amplitude of a sinusoidal function. $T$ is the period of the desired force signal expressed in sample time and $F_{0}$ is the initial desired force value. To minimize the force error $e_{f}=s_{f}-s_{f}^{*}$ (with $s_{f}$ being the measured force by a force sensor along the $y$-axis), an exponential decrease of $e_{f}$ was achieved by imposing the desired error variation of the error such as $\dot{e}_{f}^{*}=-\lambda_{f} e_{f}$ with $\lambda_{f}$ being the force control gain. The force control law consists then to apply the following control velocity to the ultrasound probe:

$$
\mathbf{v}_{f}=\mathbf{L}_{f}^{+} \dot{e}_{f}^{*}
$$

where $\mathbf{L}_{f}$ is the interaction matrix that relates the variation of the force feature to the probe velocity tensor $\mathbf{v}=$ $\left(v_{x}, v_{y}, v_{z}, \omega_{x}, \omega_{y}, \omega_{z}\right)$ such as $\dot{s}_{f}=\mathbf{L}_{f} \mathbf{v}$ and which is given by $\mathbf{L}_{f}=\left[\begin{array}{llllll}0 & K & 0 & 0 & 0 & 0\end{array}\right]$, where $K$ is an estimation of the contact stiffness between the probe and the tissue. $\mathbf{L}_{f}^{+}$ denotes the Moore-Penrose pseudo-inverse of $\mathbf{L}_{f}$.

\section{HAPTIC FEEDBACK AND TELEOPERATION CONTROL}

We propose in this paper two operational modes that assist the clinician during USE. These modes can be activated and switched on demand by the user in order to teleoperate the utrasound probe or to feel the elasticity of the tissues. The teleoperation control mode (presented in section III-A) applies to the ultrasound probe the motion of the user introduced on the haptic device while generating continuously the automatic palpation motion required for the elastography. On the other hand, the haptic control mode (presented in section III-B) generates a force from the estimated elastogram of the observed tissue and render it through the haptic device to the user. This elastogram is estimated in a chosen region of interest (ROI) of the ultrasound image. We will not recall here the principle of the elastogram estimation since it is not the topic of this paper but we will consider in the next that it is provided by an image processing as the one we detailed in [11].

\section{A. Teleoperation control}

The teleoperation mode consists in applying to the probe frame $\mathcal{F}_{c p}$ the relative displacement of the haptic handler frame $\mathcal{F}_{h}$ (see Fig. 3) that results from the user manual motion. To measure this relative displacement, we first define the initial and the current poses of the handler with respect to the base frame $\mathcal{F}_{b}$ of the haptic device as ${ }^{b} \mathbf{P}_{h 0} \in \mathbb{S E}(3)$ and ${ }^{b} \mathbf{P}_{h} \in \mathbb{S E}(3)$, respectively. The initial pose of the handler is measured only at the initialization time when the user launched the teleoperation mode and its current pose is measured at every time. In the next, we will use the notation ${ }^{a} \mathbf{M}_{b}$ to refer to the $4 \times 4$ homogeneous matrice that describes a relative pose ${ }^{a} \mathbf{P}_{b}$ of a frame $\mathcal{F}_{b}$ with respect to a frame $\mathcal{F}_{a}$. The relative pose of the handler is therefore obtained as,

$$
{ }^{h 0} \mathbf{M}_{h}=\left({ }^{b} \mathbf{M}_{h 0}\right)^{-1 b} \mathbf{M}_{h}
$$

where the operator ${ }^{-1}$ represents the inversion of a homogeneous matrix. To apply this relative motion to the probe, we then formulate the desired pose of the probe frame to reach with respect to the robot base frame $\mathcal{F}_{b r}$ as,

$$
{ }^{b r} \mathbf{M}_{c p^{*}}={ }^{b r} \mathbf{M}_{c p 0}{ }^{c p 0} \mathbf{M}_{c p}{ }^{c p} \mathbf{M}_{c p^{*}}={ }^{b r} \mathbf{M}_{c p 0}{ }^{h 0} \mathbf{M}_{h}
$$

where ${ }^{b r} \mathbf{M}_{c p 0}$ describes the initial pose of the probe frame measured at the time when the user launched the teleoperation mode, ${ }^{c p 0} \mathbf{M}_{c p}$ corresponds to the pose of the probe frame measured at current time and expressed in the initial probe frame, and ${ }^{c p} \mathbf{M}_{c p^{*}}$ is the desired pose of the probe to reach that is expressed in its current frame. To reach this 
desired probe pose, we propose to apply the task function control approach that is usually considered in the positionbased visual servoing framework [14]. In our case, the objective of the robotic task is to minimize the relative pose of the current probe frame with respect to the desired one that is defined by:

$$
{ }^{c p^{*}} \mathbf{M}_{c p}={ }^{h 0} \mathbf{M}_{h}^{-1}{ }^{c p 0} \mathbf{M}_{c p}
$$

This minimization can be performed by considering as in [14] the following error vector (of size 6) to regulate towards zero,

$$
\mathbf{e}_{t e}=\left({ }^{c p *} \mathbf{t}_{c p}, \theta \mathbf{u}\right)
$$

where ${ }^{c p *} \mathbf{t}_{c p}$ is the translation part of $c p^{*} \mathbf{M}_{c p}$ and $\theta \mathbf{u}$ is the angle/axis parameterization for its rotation part. In order to obtain a exponential decrease of this error such that $\dot{\mathbf{e}}_{t e}=-\lambda_{t e} \mathbf{e}_{t e}$, the control law consists then in applying the following control velocity to the current frame of the probe:

$$
\mathbf{v}_{t e}=-\lambda_{t e} \mathbf{L}_{t e}^{-1} \mathbf{e}_{t e}
$$

where $\lambda_{t e}$ is a positive control gain and $\mathbf{L}_{t e}$ is the interaction matrix that links the error variation to the probe velocity such that $\dot{\mathbf{e}}_{t e}=\mathbf{L}_{t e} \mathbf{v}$ and which is given by ([14]):

$$
\mathbf{L}_{t e}=\left[\begin{array}{cc}
c p^{*} \mathbf{R}_{c p} & \mathbf{0} \\
\mathbf{0} & \mathbf{L}_{\theta \mathbf{u}}
\end{array}\right]
$$

with $c p^{*} \mathbf{R}_{c p}$ being the rotation part of $c p^{*} \mathbf{M}_{c p}$ and

$$
\mathbf{L}_{\theta_{\mathbf{u}}}=\mathbf{I}_{3}-\frac{\theta}{2}[\mathbf{u}]_{\times}+\left(1-\frac{\operatorname{sinc} \theta}{\operatorname{sinc}^{2} \frac{\theta}{2}}\right)[\mathbf{u}]_{\times}^{2}
$$

Since the control law (7) is designed to perform a full 6DOF teleoperation of the ultrasound probe, we need to limit its movement in the $y$-axis of the frame $\mathcal{F}_{c p}$ for security reasons, such that the axial force control has full priority. Indeed, force control is needed for the palpation motion task introduced in section II, but it also brings safety when combined with the teleoperation task. We propose to fuse the force control and the teleoperation task by using the redundancy control framework [15]. In our case, the highest priority task is the force control defined by the velocity control law $\mathbf{v}_{f}$ given by Eq. (2) The secondary task is the teleoperation of the ultrasound probe. In order to not disturb the first task, we define a projector operator $\mathbf{P}_{f}=\mathbf{I}_{6}-\mathbf{L}_{f}^{+} \mathbf{L}_{f}$ that projects the second task onto the null space of the first one as follow:

$$
\tilde{\mathbf{v}}_{t e}=\left(\mathbf{L}_{t e} \mathbf{P}_{f}\right)^{+}\left(\dot{\mathbf{e}}_{t e}-\mathbf{L}_{t e} \mathbf{v}_{f}\right)
$$

where $\tilde{\mathbf{v}}_{t e}$ corresponds to the teleoperation control velocity component that does not influence on the achievement of the first task. The global velocity control law fusing the two tasks is then given by:

$$
\mathbf{v}_{p}=\mathbf{v}_{f}+\tilde{\mathbf{v}}_{t e}
$$

where $\mathbf{v}_{p}$ is the ultrasound probe control velocity applied at the ultrasound probe frame $\mathcal{F}_{c p}$.

\section{B. Haptic control mode}

When the user pushes a button to switch to the haptic control mode, the teleoperation task is deactivated in the control law (11) by setting $\tilde{\mathbf{v}}_{t e}=\mathbf{0}$ in order to only apply the automatic palpation motion along the axial direction of the probe. Keeping only this motion of the probe is crucial to obtain an accurate estimation of the elastogram that will be used for the force feedback rendering since the quasi-static elastography technique needs pre- and postcompression states of the tissue that result only from a force applied along the axial direction of the probe. The haptic device is then used by the clinician to manually move a small ROI that acts like a virtual probe in the image. This virtual probe (the ROI) can be translated by the user along the image axes for exploration purpose by applying a planar motion along the $x$ and $y$ axes of the handler of the haptic device. The elastogram of the tissue is then estimated for this current ROI location and used to compute a force that will be rendered to the user along the $z$ axis of the haptic device's handler in order he can feel by pushing his hand along this direction the stiffness of the tissue observed by the virtual probe.

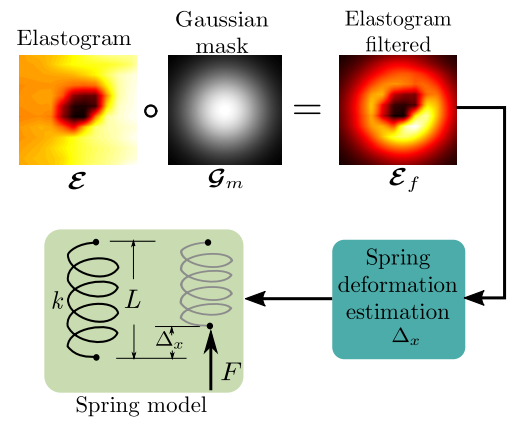

Fig. 2: Force estimation based on strain information.

1) Force estimation from elastogram: The diagram of the proposed process to estimate the force based on the elastogram observed in the ROI is shown in Fig. 2. First, the elastogram is defined as a matrix of strain values $\mathcal{E} \in$ $\mathbb{R}^{M \times N}$. As the elastogram can be affected by noise, we propose first to filter it using a Gaussian mask $\mathcal{G}_{m} \in \mathbb{R}^{M \times N}$, where the function to access every element $(i, j)$ of the Gaussian mask is defined by

$$
\bar{g}_{m}(i, j)=\frac{g_{m}(i, j)}{\max \left(\mathcal{G}_{m}\right)},
$$

where $\max \left(\mathcal{G}_{m}\right)$ is a constant value representing the maximum component value of $\mathcal{G}_{m}$, and

$$
g_{m}(i, j)=\frac{e^{-\left(\frac{i^{2}}{2 \sigma_{x}^{2}}+\frac{j^{2}}{2 \sigma_{y}^{2}}\right)}}{\sqrt{2 \pi \sigma_{x} \sigma_{y}}},
$$

where $\sigma_{x}$ and $\sigma_{y}$ are the standard deviation for lateral and axial directions, respectively. In our case, these values are typically set as $\sigma_{x}=\frac{N}{4}$ and $\sigma_{y}=\frac{M}{4}$ aiming to obtain a Gaussian distribution inside a rounded area. The center of the rounded area is located at the center of $\mathcal{G}_{m}$. Following the diagram of Fig. 2, the filtering of the elastogram is performed using $\mathcal{E}_{f}=\mathcal{E} \circ \mathcal{G}_{m}$, where $\circ$ is the Hadamard product operator and $\mathcal{E}_{f}$ is the resulting elastogram after filtering.

Next, the average scalar strain value $\varepsilon$ of $\mathcal{E}_{f}$ is computed and used in the process to generate a force $F$ that will be rendered to the user. According to the elastic Hooke's law, the reactive force generated by an elastic material is given 
by:

$$
F=-A \sigma
$$

where $A$ is the cross-sectional area of the elastic material where is applied the stress

$$
\sigma=E \varepsilon
$$

with $E$ being the Young's modulus of the material (modulus of elasticity) and $\varepsilon$ is the observed strain of the material. In the haptic control mode we propose to render a force to the user along the $\mathrm{z}$ translation axis of the haptic handler by implementing a virtual spring of length $L$ (see Fig. 2) that will reflect the stiffness $k$ of the tissue sample observed at the center of the ROI. Following the Hooke's law, the reactive force generated by a spring that is compressed by a displacement $\Delta x$ is given by:

$$
F=-k \Delta_{x} \quad \text { with } \quad \Delta_{x}=\varepsilon L
$$

The determination of the stiffness parameter $k$, that reflects the tissue stiffness of the tissue sample located at the center of the ROI, consists then in substituting Eq. (15-16) in Eq. (14) with $\varepsilon$ being the scalar strain value estimated from the ROI, and it gives:

$$
k=\frac{A E}{L}
$$

where $A$ is the region area of the virtual probe that senses the local strain of the tissue in the ROI and that corresponds in our case to the elliptical surface $A=\pi \sigma_{x} \sigma_{y}$ of the Gaussian mask $\mathcal{G}_{m} . E$ is the Young's modulus that we set to the value $E=3 k P a$ of healthy tissue and $L$ is the original length of the virtual spring that corresponds to the width $d$ of the rectangular contact surface between the real ultrasound probe and the tissue (see Fig. 1b).

2) Virtual probe control and force feedback: Fig. 3 illustrates the principle that consists in moving the ROI to follow the displacement of the user measured by the handler of the haptic device. If the user applies motion at the handler of the haptic device $\mathcal{F}_{h}$, then the center of the ROI $\left(u_{c}, v_{c}\right)$ is shifted with a displacement $\Delta_{d} \in \mathbb{R}^{2}$ proportional to the displacement of the handler such that,

$$
\Delta_{d}=\mathbf{S} \Delta_{h}
$$

where $\Delta_{h} \in \mathbb{R}^{2}$ is the in-plane relative motion applied to the handler that is directly measured from the $\mathrm{x}$ and $\mathrm{y}$ translation components of the $4^{\text {th }}$ column of the homogeneous matrix ${ }^{h 0} \mathbf{M}_{h}$ introduced in eq. (3). $\mathbf{S}=\operatorname{diag}\left(s_{x}, s_{y}\right) \in \mathbb{R}^{2 \times 2}$ is a $2 \times 2$ diagonal matrix containing the scale values $\left(s_{x}, s_{y}\right)$ that convert pixels to meters for lateral and axial direction.

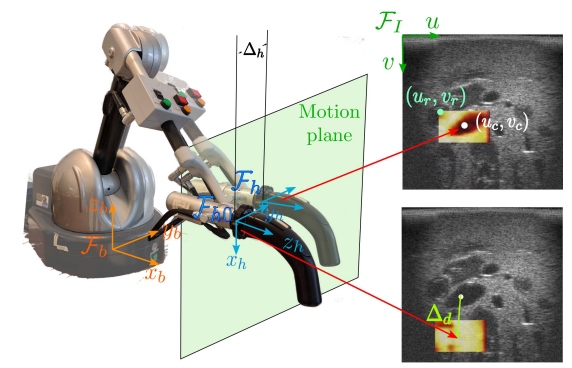

Fig. 3: Application of the handler in-plane displacement to manually move the ROI where is estimated the elastogram in the ultrasound image.

The displacement $\Delta_{d}$ of the ROI generates a new elas- togram which is translated to a force feedback $F$ using the method described in section III-B.1 through Eq. (16). This force value is then applied to the low-level force impedance control scheme of the haptic device along the $z$ axis of its handler in order the user feels the effect of the virtual spring that reflects the elasticity of the elastogram when he pushes the handler in the $z$ direction of $\mathcal{F}_{h}$. At the same time, he can also move the virtual probe (ROI) in the ultrasound image by applying a planar motion along the $x-y$ axes of the handler frame $\mathcal{F}_{h}$ and feel along the $z$ direction the force generated by the current elastogram displayed in the new ROI location.

\section{EXPERIMENTAL RESULTS}

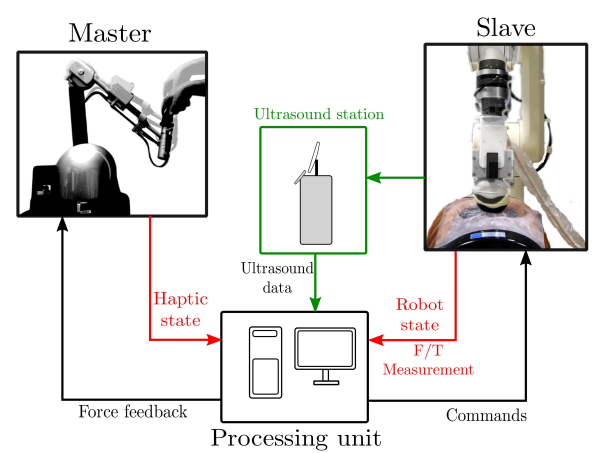

Fig. 4: Experimental setup of the proposed haptic system.

This section presents the results of the teleoperation and haptic feedback system previously described. First, we define the experimental setup as illustrated in Fig. 4. The haptic device used for the experiments is the Virtuose 6D (Haption S.A.). We used a 6-DOF robot Viper s850 (Adept Technology Inc., USA) equipped with a force/torque sensor ATI Gamma 65-SI FT plugged to a National Instrument NI DAQmx PCI6220 board. We also attached to the robot end-effector a convex ultrasound probe (Analogic Corporation 4DC7-3/40) that we used in 2D imaging mode. An abdominal phantom ABDFAN US-1B containing artificial soft tissues and several rigid cysts was employed for our tests.

The haptic system measures the current handler pose with an update rate frequency of $100 \mathrm{~Hz}$, and the new relative displacement $\Delta_{d}$ is sent to the elastography process to change the position of the ROI. Two buttons located on the handler allow the user to switch between the impedance force feedback and the teleoperation control modes. When the teleoperation process is activated, the velocities are applied to the robot by using the Eq.(11). The robot is continuously applying the oscillation in the $y$-axis force needed to obtain the pre- and post-compressed states of the tissues required for the elastogram estimation. The elastogram is displayed in a graphical user interface to provide the visualization of the ROI.

In the next, we present the results of an experiment that consists of two parts. First, the initial state of the system is in teleoperation mode, and the user can explore the tissue by moving the handler of the haptic device. Then, the user switches from the teleoperation mode to the haptic force feedback mode. 


\section{A. Teleoperation results}

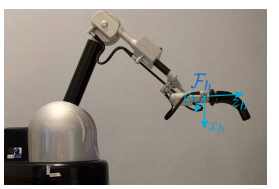

(a) Haptic state at the (b) Handler rotation in tem.
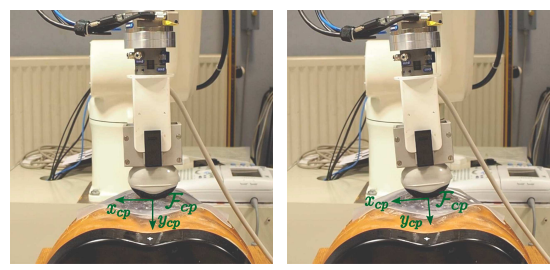

(d) Probe reaching the contact force.

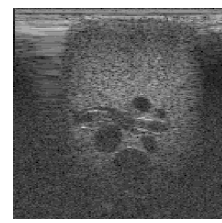

(g) initialization of the sys- $z$-axis.
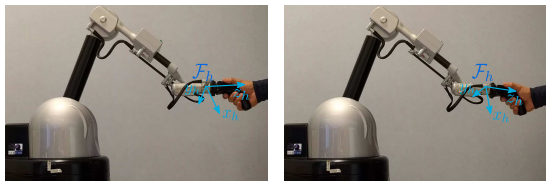

(c) Handler with an arbitrary pose.

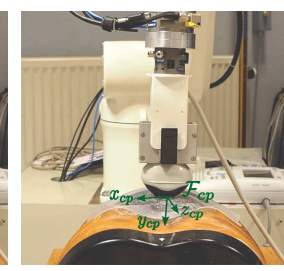
(e) Probe pose for the (f) Probe pose for the
handler pose at $(5 b)$. handler pose at $(5 c)$.

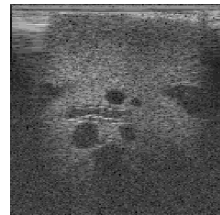

(h)

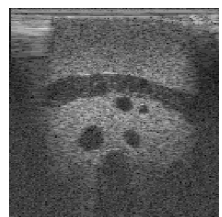

(i)
Fig. 5: Teleoperation system states. (a) initial pose of the handler and (d) the corresponding pose of the ultrasound probe with the $(\mathrm{g})$ resulting ultrasound image. (b) rotation around $z$-axis of the handler and (e) the corresponding pose of the ultrasound probe with the (h) resulting ultrasound image. (c) arbitrary pose of the handler and (f) the corresponding pose of the ultrasound probe with the (i) resulting ultrasound image.

Fig. 5 shows in the first row different configurations of the haptic device when the user applied manual motion on the handler during the teleoperation control mode. The second row of Fig. 5 presents the resulting pose of the robot holding the probe and the third row provides the observed ultrasound image for each configuration. Fig. 6 shows the temporal evolution of the measured force and the control velocities applied to the probe. The palpation motion task is activated, initiating the force control to reach contact with the phantom as shown in Fig. 5d. Fig. 6a shows at $t=\sim 2.5 \mathrm{~s}$ the beginning of the force variation needed for the palpation motion. The teleoperation of the US probe with the haptic device starts at $t=\sim 13 \mathrm{~s}$ as indicated with the black arrow in the plot illustrating the evolution of the teleoperation errors from $t=10 \mathrm{~s}$ to $t=52 \mathrm{~s}$ (Fig. 6c). At the same time, we can also observe in Fig. $6 \mathrm{~b}$ that the velocities applied to the ultrasound probe related to the teleoperation task $\left(v_{x}, v_{z}, \omega_{x}, \omega_{y}\right.$ and $\left.\omega_{z}\right)$ start to variate in order to replicate the motion introduced by the user in the handler of the haptic device. After, in Fig. 6c we highlighted two moments when the user was applying continuous motion to the haptic device (strips in light-blue color) and when the user stops the motion (strips in light-green color). We can observe the fast convergence $(\sim 0.5 \mathrm{~s})$ of the teleoperation system in both cases. In these plots, one can observe the variation in the velocities and the errors due to the different motions introduced to the handler of the haptic device. The parameters of the desired force variation were set to $5 \mathrm{~N}$ and $3 \mathrm{~N}$ for the minimum and variation forces, respectively. We can observe that the measured force follows correctly the desired oscillation reference, since the force control task that has the highest priority is not disturbed by the teleoperation task.

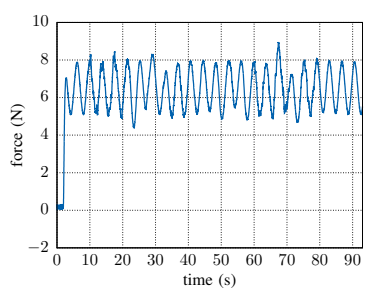

(a) Force

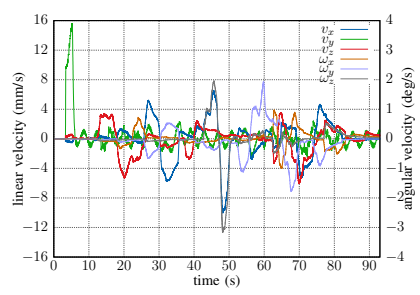

(b) Probe linear velocities.

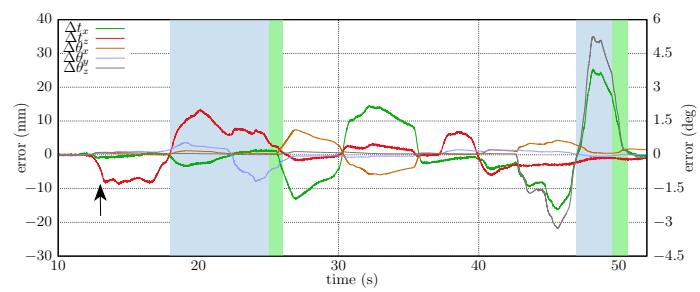

(c) Teleoperation errors.

Fig. 6: Measured force, velocities and errors in the teleoperation system.

\section{B. Haptic feedback results}

The haptic feedback control mode starts after selecting a ROI where the elastogram is estimated in real time, the user can feel the force computed from the elastogram while moving the handler of the haptic device. We present the results of one experiment where the user moves the virtual probe position, corresponding to the ROI, inside the ultrasound image with the haptic device. We can observe in Fig. 7 the plot that represents the haptic force feedback applied to the handler of the haptic device along its $z$ axis direction. The ultrasound images with the elastogram overlaid are placed for some states along the force feedback evolution to show the position of the elastogram. Above every ultrasound image we display the image of the current state of the haptic device. We can notice the higher forces applied to the handler of the haptic device when stiff tissues (dark areas) are observed in the ROI representing the teleoperated virtual probe.

The confidence in the force feedback measurements while moving the virtual probe is analyzed by performing repeated force value estimations along a trajectory of the virtual probe represented by the green path shown in Fig. 8. The test consists in measuring the force feedback for 110 positions along the green path with a position changing rate of 40 FPS (total duration of 2.4s). The same motion is then repeated 50 times while the impedance force feedback mode is running to statistically measure the average of the force feedback for different locations of the ROI. Through this test, we noticed small variations in the average of the force feedback. Fig. 9 shows the force feedback average after the 50 repetitions of the green path, where the black line is the force feedback average and green area represents the interquartile range 

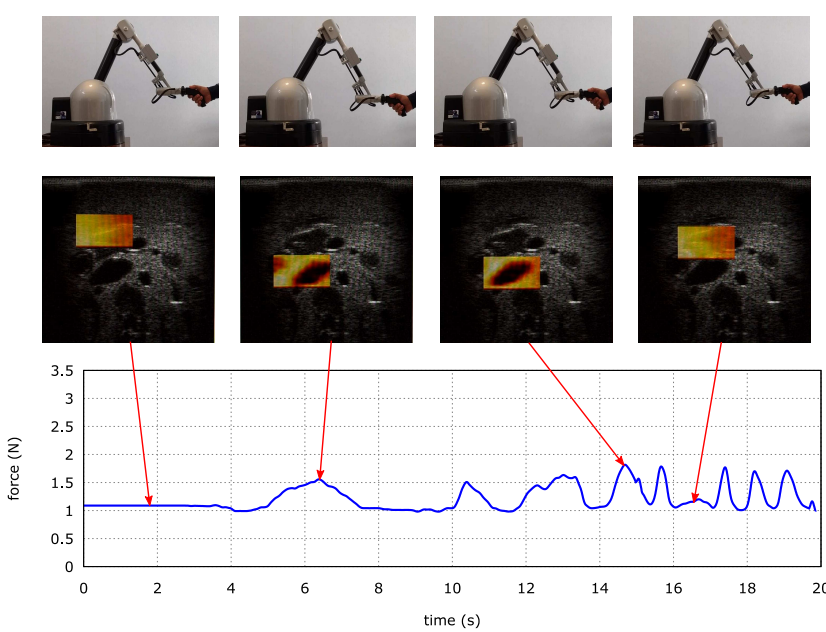

Fig. 7: Result of the force feedback of the impedance system. First row shows the different states while moving the handler of the haptic device. The motion of the ROI containing the elastogram is shown in the second row for the different states of the handler motion. The temporal evolution of the force feedback applied on the $z$ axis of the haptic device's handler is plotted at the bottom and the position of the states are indicated with red arrows.

(IQR). The small IQR for all the positions shown in the plot of Fig. 9 describes the standard deviation (SD). Based on the observed small variation (maximum SD of $0.21 \mathrm{~N}$ ) of the force feedback after 50 repetitions of the green path illustrated in Fig. 8, we can conclude that our force feedback measurement in the ROI is highly reproducible.
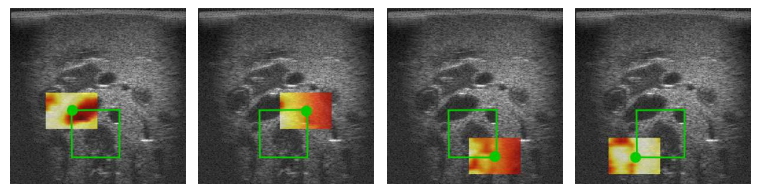

Fig. 8: Repetitive motion path of the virtual probe. The four images correspond to the four corners of the square path. The green path was used to measure the standard deviation of the force feedback.

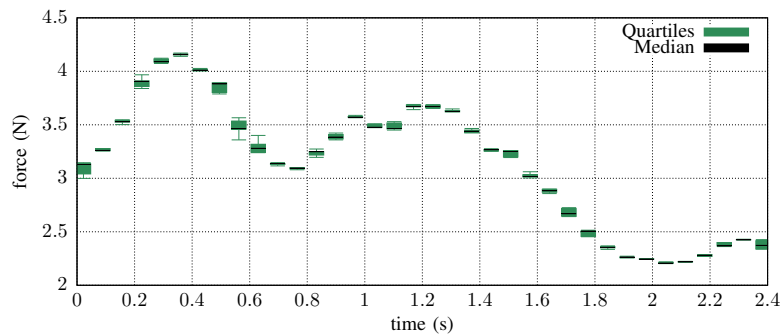

Fig. 9: Force feedback average after 50 repetitions of the squared path shown in Fig. 8.

\section{CONCLUSions}

Two assistance modes were proposed in this work to aid the examiner to perform USE of the tissue and to simultaneously feel the elasticity of the tissue with a haptic device. This system with the teleoperation of a 2D ultrasound probe offers the capabilities to remotely perform USE on a patient or simply to confirm the tissue elasticity displayed in the elastogram. We have demonstrated experimentally a good performance for both teleoperation and impedance haptic control modes. The force feedback using the elastogram was statistically evaluated to determine its reproducibility. However, the estimation of the force feedback from the elastogram assumes a specific Young's modulus of the soft tissue and this may variate between different kinds of tissues. Despite of the coarse assumption value of the Young's modulus, the force feedback feeling obtained from the experimental results is promising, and offers the possibility to perform a future study with expert physicians to validate this force feedback functionality assistance.

\section{REFERENCES}

[1] J. J. Abbott and A. M. Okamura, "Virtual fixture architectures for telemanipulation," in IEEE International Conference on Robotics and Automation, vol. 2, Sept 2003, pp. 2798-2805.

[2] A. M. Okamura, "Methods for haptic feedback in teleoperated robotassisted surgery," Industrial Robot: the international journal of robotics research and application, vol. 31, no. 6, pp. 499-508, 2004.

[3] S.-Y. Kim, J.-H. Ryu, and W. Lee, "Palpation simulator with stable haptic feedback," Minimally Invasive Therapy \& Allied Technologies, vol. 24, no. 4, pp. 211-217, dec 2014.

[4] S. McKinley, A. Garg, S. Sen, R. Kapadia, A. Murali, K. Nichols, S. Lim, S. Patil, P. Abbeel, A. M. Okamura, and K. Goldberg, "A single-use haptic palpation probe for locating subcutaneous blood vessels in robot-assisted minimally invasive surgery," in 2015 IEEE International Conference on Automation Science and Engineering, August 2015, pp. 1151-1158.

[5] L. Meli, C. Pacchierotti, and D. Prattichizzo, "Experimental evaluation of magnified haptic feedback for robot-assisted needle insertion and palpation," The International Journal of Medical Robotics and Computer Assisted Surgery, vol. 13, no. 4, pp. e1809-n/a, 2017.

[6] M. Li, J. Konstantinova, E. L. Secco, A. Jiang, H. Liu, T. Nanayakkara, L. D. Seneviratne, P. Dasgupta, K. Althoefer, and H. A. Wurdemann, "Using visual cues to enhance haptic feedback for palpation on virtual model of soft tissue," Medical \& Biological Engineering \& Computing, vol. 53, no. 11, pp. 1177-1186, November 2015.

[7] J. L. Preston, T. M. Byun, S. E. Boyce, S. Hamilton, M. Tiede, E. Phillips, A. Rivera-Campos, and D. H. Whalen, "Ultrasound images of the tongue: A tutorial for assessment and remediation of speech sound errors," Journal of Visualized Experiments, no. 119, jan 2017.

[8] A. Goddi, M. Bonardi, and S. Alessi, "Breast elastography: A literature review," Journal of Ultrasound, vol. 15, no. 3, pp. $192-$ 198, 2012.

[9] R. G. Barr, G. Ferraioli, M. L. Palmeri, Z. D. Goodman, G. GarciaTsao, J. Rubin, B. Garra, R. P. Myers, S. R. Wilson, D. Rubens, and D. Levine, "Elastography assessment of liver fibrosis: Society of radiologists in ultrasound consensus conference statement," Radiology, vol. 276, no. 3, pp. 845-861, 2015.

[10] J.-M. Correas, A.-M. Tissier, A. Khairoune, G. Khoury, D. Eiss, and O. Hlnon, "Ultrasound elastography of the prostate: State of the art," Diagnostic and Interventional Imaging, vol. 94, no. 5, pp. 551 - 560, May 2013.

[11] P. A. Patlan-Rosales and A. Krupa, "Automatic palpation for quantitative ultrasound elastography by visual servoing and force control," in IEEE/RSJ Int. Conf. on Intelligent Robots and Systems (IROS), Daejeon, 2016.

[12] J. Konstantinova, M. Li, V. Aminzadeh, K. Althoefer, Thrishantha, and P. Dasgupta, "Evaluating manual palpation trajectory patterns in tele-manipulation for soft tissue examination," in 2013 IEEE International Conference on Systems, Man, and Cybernetics, 2013, pp. 4190-4195.

[13] K. L. Palmerius, R. F. Havre, O. H. Gilja, and I. Viola, "Ultrasound palpation by haptic elastography," in 2011 24th International Symposium on Computer-Based Medical Systems, June 2011, pp. 1-6.

[14] F. Chaumette and S. Hutchinson, "Visual servo control, part I: Basic approaches," IEEE Robotics and Automation Magazine, vol. 13, no. 4, pp. 82-90, December 2006.

[15] B. Siciliano and J.-J. Slotine, "A general framework for managing multiple tasks in highly redundant robotic systems," in Fifth International Conference on Advanced Robotics., 1991, pp. 1211-1216. 